\title{
Estudios experimentales sobre la consolidación y protección de los materiales calcáreos de la Catedral de Oviedo. 1. ${ }^{\mathrm{a}}$ parte
}

\author{
Laboratory studies on the consolidation \\ and protection of calcareous materials \\ in the Cathedral of Oviedo. Part I
}

ROSA M. ${ }^{\text {a }}$ ESBERT, CARLOTA GROSSI y ROSA M. MARCOS Area de Petrología. Dpto. de Geologia. Universidad de Oviedo

\section{RESUMEN}

Se presenta una metodología de trabajo encaminada a evaluar la idoneidad de ciertos productos consoliantes y protectores aplicados a piedras monumentales deterioradas. Para ello, en esta $1 .^{a}$ parte, se determinan una serie de propiedades físicas, antes y después de aplicar los tratamientos. Dichas propiedades han sido seleccionadas por su incidencia en los mecanismos de alteración.

Las piedras ensayadas proceden de la Catedral de Oviedo. son rocas calcáreas: calizas y dolomias. Los productos de tratamiento empleados han sido: silicato de etilo, silano oligomérico y resina silicónica.

Se concluye con la comparación de las variaciones en las propiedades determinadas, antes y después del tratamiento. según el producto empleado.

\section{SUMMARY}

The suitability of some consolidating and protecting products, to be applied to the deteriorated calcareous stones of the Cathedral of Oviedo, is presented. A proposed procedure and methodology for evaluating that suitability is also described.

The studied stones, limestones and dolomites, have been experimentally subjected in laboratory to some treatment products: ethyl silicate, oligomeric silane and silicon resin.

Some physical properties of those stones were evaluated before and after the treatments with the mentioned products. Those properties (porosity, water absorption, compressive strength and deformability, mechanical microfissuration threshold, acoustic emission, etc.) were selected according to the significative role they play on the process of stone alteration or in supporting the propossed conclusions.

\section{INTRODUCCION}

En numerosas edificaciones del Patrimonio Arquitectónico español, el tipo y grado de deterioro alcanzado por sus piedras de construción hacen plantear, como una de las fases del proceso de restauración, la consolidación y protección de las mismas (Amoroso y Fassina, 1983).

Este es el caso de numerosas partes de la fábrica gótica de la Catedral de Oviedo, ubicada en un ambiente particularmente agresivo (urbano-húmedo) que ha propiciado que sobre sus piedras de edificación se generen formas de alteración de índole

\section{INTRODUCTION}

Consolidating and protecting the building stones in many Monuments of the Architectonic National Heritage of Spain forms part of their restoration process. These solutions are undertaken according to the previous studies on the types and degrees of deterioration reached by the stones in a given Monument.

This is the case of many parts of the building stones of the Gothic Cathedral of Oviedo, located in a particularly aggressive, urban, humid environment which has favoured the appearance of different alteration forms which, finally, cause loss of cohesion among the stone 
diversa, las cuales, en última instancia, provocan la pérdida de cohesión de los componentes de la piedra (Esbert y Marcos, 1983).

Este estudio pretende documentar, en su 1. parte, el efecto de tres productos orgánicos con características consolidantes e hidrorrepelentes, aplicados a los materiales calcáreos de la Catedral de .Oviedo.

En la 2. ${ }^{a}$ parte se evaluará, mediante ensayos de envejecimiento acelerado, la durabilidad de las piedras tratadas.

\section{MATERIALES PETREOS Y PRODUCTOS DE TRATAMIENTO}

Las piedras de edificación tratadas han sido la dolomía de Laspra de edad peleógena y la caliza de Piedramuelle del Cretácico Superior. Ambas representan los tipos petrográficos mayoritariamente empleados en la Catedral (Esbert et al., 1982).

Los productos de tratamiento elegidos persiguen, tanto la consolidación de las piedras - hacerlas más resistentes mecánicamente-, como la protección de las mismas, confiriéndoles un carácter hidrorrepelente, que las convierta en más resistentes a los agentes alterantes, agua fundamentalmente.

En concreto los productos de tratamiento utilizados han sido:

- Silicato de etilo -SE-, que actúa como consolidante y silano oligomérico - SO-, que actúa como protector.

- Resina silicónica -RS-, que actúa simultáneamente como consolidante y protector.

Tanto el SE como el SO, son de la firma comercial Wacker Chemie (República Federal de Alemania), recibiendo respectivamente los nombres de $\mathrm{OH}$ Wacker y $290 \mathrm{~L}$ Wacker.

La RS es de la firma Rhône-Poulenc (Francia) conociéndose con el nombre de Rhodorsil 11309

El OH Wacker viene preparado para su utilización inmediata, no precisando una dilución previa.

El 290L Wacker y el Rhodorsil 11309 se han aplicado disueltos en $1: 1: 1$ tricloroetano.

La elección y modo de aplicación de estos components (Esbert and Marcos, 1983).

Laboratory experiments were carried out, to evaluate the effects of three organic products -their consolidating and hydrophobic properties - when applied to the calcareous materials of the Cathedral of Oviedo.

\section{STONE MATERIALS AND TREATMENT PRODUCTS}

The studied building stones were Laspra dolomite (Paleogenic) and Piedramuelle limestone (Upperior Cretaceus), both representig the most common stones used in the construction of the Cathedral (Esbert et al. 1982).

The selected treatment products pursue not only the consolidation of stones - by increasing their mechanical strength- but also their protection, giving to the stones an hydrophobic character which will make them more durable to the alteration agents, fundamentaily water.

Specifically, the treatments used were:

- Ethyl silicate -ES - working as a consolidant, and oligomeric silane -OSwhich acts as a protector.

- Silicon Resin - SR-working simultaneously as consolidant and protector.

Both ES and OS used are Wacker Chemie products (Federal Republic of Germany), receiving the names of $\mathrm{OH}$ Wacker and $290 \mathrm{~L}$ Wacker, respectively.

SR is produced by Rhône-Poulenc (France), being its commercial name Rhodorsil 11309.

$\mathrm{OH}$ Wacker is a ready-made product which does not need previous dilution.

290L. Wacker and Rhodorsil 11309 were used in a solution of 1:1:1 Trichlorethane.

Those products were selected according to 
productos ha contado con el asesoramiento del Istituto Centrale del Restauro (Roma) que ha venido aplicándolos en diversos monumentos italianos, sobre piedras de características similares a las de la Catedral de Oviedo, con resultados satisfactorios.

\section{MODO DE APLICACION}

Para la aplicación de los tratamientos en el laboratorio se han tallado bloques de piedra procedentes de la Catedral, dándoles la forma más regular posible. Sus dimensiones aproximadas han sido de $30 \times 10 \times 10 \mathrm{~cm}$. Previa a la apiicación de los tratamientos se ha realizado la limpieza de los bloques, mediante diferentes métodos (agua nebulizada, microbaño de arena, etc.).

Posteriormente se han envuelto las piedras en algodó hidrófilo, sujeto con vendas, para asegurar un total contacto entre el algodón y la piedra.

La aplicación de los productos se ha realizado utilizando un sistema gota a gota que ha ido impregnando lentamente (durante cinco días) el algodón en contacto con la roca. De esta manera se consigue que el producto quede uniformemente distribuido por su superficie (Fig. 1) (Grossi, 1986). previous experiences carried out at Istituto Centrale del Restauro (Roma); they were satisfactorily applied to some Italian Monuments which stones were very similar to those in the Cathedral of Oviedo.

\section{PROCEDURES}

Specimens from some blocks of approximately $30 \times 10 \times 10 \mathrm{~cm}$ in size from the Cathedral were prepared for the laboratory experiments. Before the applying the treatment, the blocks were cleaned by means of several methods (nebulized water, microblasting, etc.).

The application of the products was performed by a drop-by-drop method, that slowly impregnates (during five days) the cotton around the rock. So, the product was uniformly distributed over its surface (Fig. 1)

(Grossi, 1986).

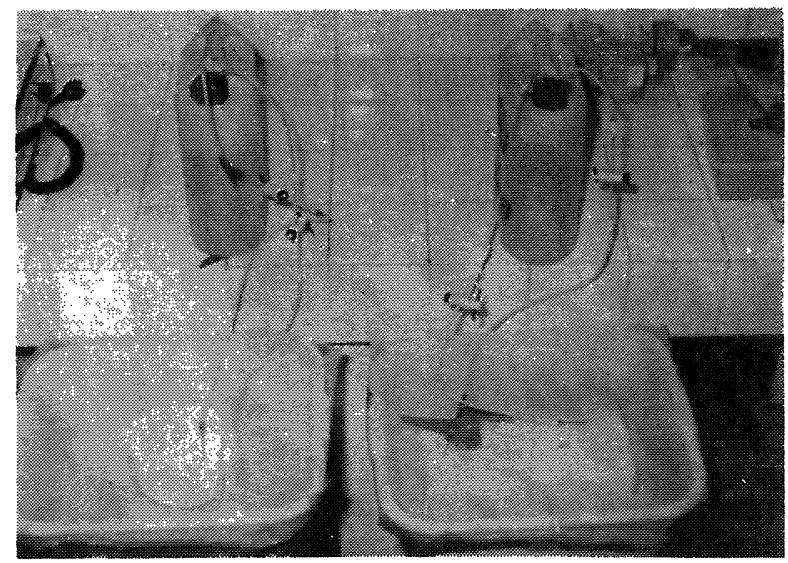

Fig. 1.-Dispositivo gota a gota para la aplicación de los tratamientos.

\section{DETERMINACION DE PROPIEDADES QUE CARACTERIZAN LA EFICACIA DEL TRATAMIENTO}

Para establecer criterios sobre el efecto de los productos de tratamiento, se han determinado, antes y después de su aplicación, ciertas propiedades relacionadas con los procesos de alteración, así como con la durabilidad de los materiales; tal es el caso de las propiedades
Fig. 1.-Drop-by-drop method for treatment application.

\section{PROPERTIES CHARACTERIZING THE TREATMENT EFFICIENCY}

Some physical properties, related with alteration processes and the durability of materials, have been determined in order to establish criteria for evaluating the effects of the treatments before and after their application. Special emphasis has been 
hídricas, relacionadas con la circulación del agua por el seno de los mismos (Esbert et al., 1987.

Las propiedades determinadas han sido:

- Porosidad abierta (no): distribución porométrica.

- Contenido en humedad de saturación (Ws).

- Velocidad de absorción libre de agua por inmersión y coeficiente de absorción (W).

- Velocidad de evaporación de agua.

- Succión capilar (C).

- Resistencia a la compresión uniaxial (Rc) y Módulo de Young $\left(E_{\tau}\right)$.

- Registro de la emisión acústica y del umbral de microfisuración mecánica (UMM) $\left(^{*}\right)$.

Las normas seguidas para la determinación de estas propiedades han sido las de la I.S.R.M. $(1979,1981)$ y C.N.R.-I.C.R. $(1981,1983)$. En la actualidad se están determinando otras propiedades significativas con relación a los efectos del tratamiento, tales como: color, permeabilidad al vapor y ángulo de contacto.

En las tablas I, II y III, se presentan los valores de las propiedades antes (Laspra y Piedramuelle 1) y después (Laspra y Piedramuelle 2 y 3) de los tratamientos.

En las figuras 2, 3, 4 y 5 se representan algunas de las propiedades evaluadas.

TABLA I

Valores de la porosidad y distribución porométrica obtenidos mediante porosimetría de mercurio, de las piedras de la Catedral de Oviedo; 1 muestras sin tratar; 2 muestras tratadas con RS: 3 muestras tratadas con $S E+S O$

\begin{tabular}{|l|c|c|c|c|c|}
\hline & $\begin{array}{c}\text { no } \\
(\%)\end{array}$ & $\begin{array}{c}\text { Porosid. } \\
\text { atrapada } \\
(\%)\end{array}$ & $\begin{array}{c}\text { Porosid. } \\
\text { libre } \\
(\%)\end{array}$ & $\begin{array}{c}\text { Macro } \\
\text { porosid. } \\
(\%)\end{array}$ & $\begin{array}{c}\text { Micro } \\
\text { porosid. } \\
(\%)\end{array}$ \\
\hline Laspra 1 & 31.4 & 20,0 & 11,4 & 0 & 31,4 \\
Laspra 2 & 24.0 & 13,5 & 10,5 & 0 & 24,0 \\
Laspra 3 & 22,5 & 14,0 & 8,5 & 0 & 22,5 \\
\hline Piedram. 1 & 19,0 & 8,6 & 7,5 & 2,9 & 16,1 \\
Piedram. 2 & 14.0 & 9,0 & 4,6 & 0,4 & 13,6 \\
Piedram. 3 & 14,4 & 11,0 & 3,2 & 0,2 & 14,2 \\
\hline
\end{tabular}

(*) UMM: \% de resistencia a la que se inicia la microfisuración del material. conferred to hydric properties, related with the water sorption and circulation through the interior of the stones (Esbert et al., 1987)

The properties determinest were:

- Open porosity (no): porometric distribution.

- Water saturation content (Ws).

- Water absorption rate through immersion and absorption coefficient (W).

- Water desorption rate.

- Capillary suction (C).

- Uniaxial compressive strength (Rc) and Young's modulus ( $\left.E_{\tau}\right)$.

- Acoustic emission under loading and mechanical microfissuration threshold $(M M T)(*)$.

The procedures followed for determining these properties were the ISRM suggested methods (1979; 1981) and the CNR-ICR $(1981,1983)$. Other significant properties related to the treatment evaluation were also determined, in the Part II such as: weight, colour, water vapour permeability, contact angle,...

Tables I, II and III show the values of the properties measured before (Laspra and Piedramuelle 1) and after (Laspra and Piedramuelle, 2 and 3) the reatments.

Figures 2, 3, 4 and 5 represent some of the measured properties.

\section{TABLE I}

Porosity values and porometric distribution for the stones of the Cathedral of Oviedo, obtained by means of mercury porosimetry. 1: non treated samples; 2: samples treated with SR; 3: samples treated with ES and OS

\begin{tabular}{|l|c|c|c|c|c|}
\hline & $\begin{array}{c}\text { "Open"" } \\
\text { porosity } \\
\text { (\%) }\end{array}$ & $\begin{array}{c}\text { "Trapped" } \\
\text { porosity } \\
\text { (\%) }\end{array}$ & $\begin{array}{c}\text { "Free"” } \\
\text { porosity } \\
\text { (\%) }\end{array}$ & $\begin{array}{c}\text { Macro } \\
\text { porosity } \\
(\%)\end{array}$ & $\begin{array}{c}\text { Micro } \\
\text { porosity } \\
\text { (\%) }\end{array}$ \\
\hline Laspra 1 & 31,4 & 20,0 & 11,4 & 0 & 31,4 \\
Laspra 2 & 24.0 & 13,5 & 10,5 & 0 & 24.0 \\
Laspra 3 & 22,5 & 14,0 & 8,5 & 0 & 22.5 \\
\hline Piedram. 1 & 19.0 & 8,6 & 7,5 & 2,9 & 16.1 \\
Piedram. 2 & 14.0 & 9,0 & 4,6 & 0.4 & 13.6 \\
Piedram. 3 & 14,4 & 11,0 & 3,2 & 0.2 & 14.2 \\
\hline
\end{tabular}

${ }^{\star}$ ) The stress-level, expressed as a porcentage of the ultimate strength, at which microfissuration begin to grow). 
TABLA II

Valores de absorción libre de agua por inmersión (W); contenido en humedad de saturación (Ws) y coeficiente de capilaridad (C) de las piedras de la Catedral de Oviedo. 1 muestras sin tratar; 2 muestras tratadas con RS y 3 muestras tratadas con $S E+S O$

\begin{tabular}{|l|c|c|c|}
\hline & $\begin{array}{c}\mathbf{W} \\
(\%)\end{array}$ & $\begin{array}{c}\text { Ws } \\
(\%)\end{array}$ & $\begin{array}{c}\mathbf{C} \\
\left(\mathbf{k g} / \mathbf{m}^{2} \mathbf{m i n}^{\mathbf{0 . 5}}\right.\end{array}$ \\
\hline Laspra 1 & 13,6 & 16,0 & 60,0 \\
Laspra 2 & 9,8 & 14,0 & 27,0 \\
Laspra 3 & 8,2 & 10,0 & 16,5 \\
\hline Piedram. 1 & 7,7 & 10,0 & 36,5 \\
Piedram. 2 & 3,7 & 7,4 & 3,0 \\
Piedram. 3 & 4,3 & 6,0 & 7,0 \\
\hline
\end{tabular}
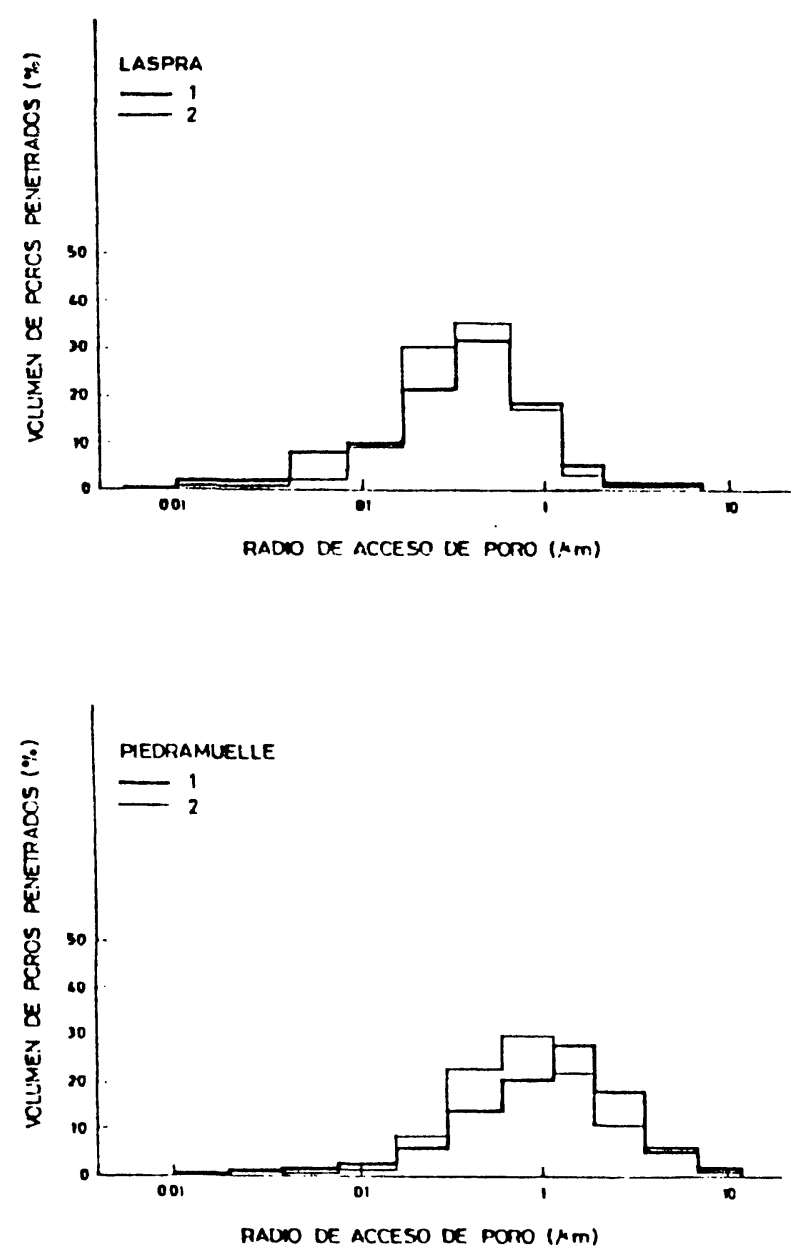

Fig. 2.- Histogramas con la distribución porométrica de las variedades rocosas de Laspra y Piedramuelle. 1 muestras no tratadas; 2 muestras tratadas con RS y 3 muestras tratadas con SE + SO. En ellas se visualiza también el volumen de mercurio penetrado para cada rango de radio de acceso de poro.
TABLE II

Water free absorption values (W); Water saturation content (Ws), and Capillarity coefficient $(C)$ for the stones of the Cathedral of Oviedo. 1: non treated samples; 2: samples treated with SR; 3: samples treated with ES and OS

\begin{tabular}{|l|c|c|c|}
\hline & $\begin{array}{c}\text { W } \\
(\%)\end{array}$ & $\begin{array}{c}\text { Ws } \\
(\%)\end{array}$ & $\begin{array}{c}\mathbf{C} \\
\left(\mathbf{k g} / \mathbf{m}^{2} \mathbf{~ m i n}^{\mathbf{0 . 5}}\right.\end{array}$ \\
\hline Laspra 1 & 13,6 & 16,0 & 60,0 \\
Laspra 2 & 9,8 & 14,0 & 27.0 \\
Laspra 3 & 8,2 & 10,0 & 16,5 \\
\hline Piedram. 1 & 7,7 & 10,0 & 36,5 \\
Piedram. 2 & 3,7 & 7,4 & 3,0 \\
Piedram. 3 & 4,3 & 6,0 & 7.0 \\
\hline
\end{tabular}
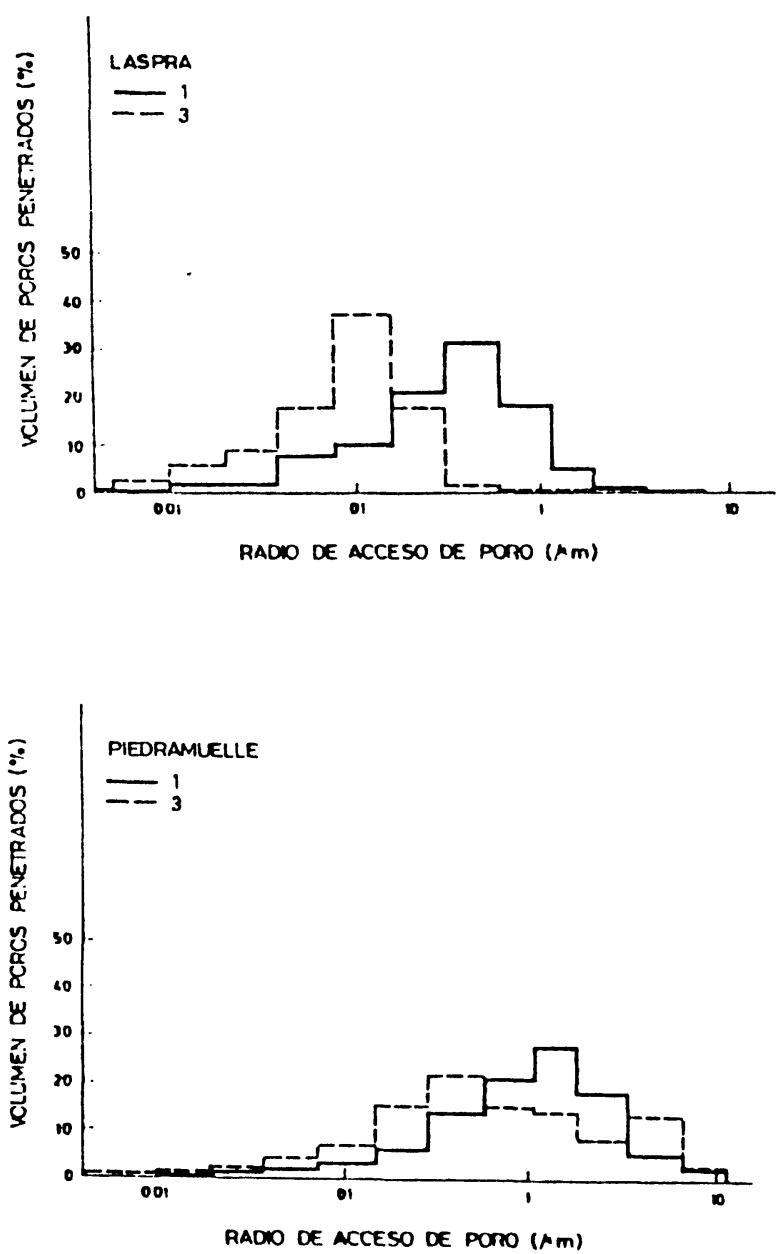

Fig. 2.-Porometric distribution of the rock varieties from Laspra and Piedramuelle. 1: non-treated samples: 2: samples treated with SR; and 3: samples treated with ES and OS. The volume of intruded mercury for each porerange is also visualized. 
TABLA III

Resultados obtenidos a partir del ensayo de compresión uniaxial, para la dolomía de Laspra; 1 sin tratar; 2 tratados con RS y 3 tratada con $S E+S O$

\begin{tabular}{|l|c|c|c|c|}
\hline & $\begin{array}{c}\mathbf{R c} \\
(\mathbf{M P a})\end{array}$ & $\begin{array}{c}\varepsilon_{1} \\
(\mu \delta)\end{array}$ & $\begin{array}{c}\mathbf{E} \\
(\mathbf{M P a})\end{array}$ & $\begin{array}{c}\text { UMM } \\
(\%)\end{array}$ \\
\hline Laspra 1 & 23,6 & 2.000 & $3.0 \times 10^{4}$ & 50 \\
Laspra 2 & 34,0 & 3.600 & $1,0 \times 10^{4}$ & 75 \\
Laspra 3 & 52,0 & 4.200 & $1,2 \times 10^{4}$ & 67 \\
\hline
\end{tabular}

$R_{C} \quad$ - Resistencia a la compresión uniaxial.

$\varepsilon_{\mid} \quad=$ Deformación longitudinal

UMM - - Umbral de microfisuración mecánica.
TABLE III

Uniaxial compressive strength values for the Laspra Dolomite. 1: non-treated; 2: treated with SR; 3: treated with ES and OS

\begin{tabular}{|l|c|c|c|c|}
\hline & $\begin{array}{c}\mathbf{R}_{\mathbf{c}} \\
(\mathbf{M P a})\end{array}$ & $\begin{array}{c}\varepsilon_{1} \\
(\mu \delta)\end{array}$ & $\begin{array}{c}\mathbf{E}_{\mathbf{}} \\
(\mathbf{M P a})\end{array}$ & $\begin{array}{c}\text { UMM } \\
(\%)\end{array}$ \\
\hline Laspra 1 & 23,6 & 2.000 & $3.0 \times 10^{4}$ & 50 \\
Laspra 2 & 34,0 & 3.600 & $1.0 \times 10^{4}$ & 75 \\
Laspra 3 & 52,0 & 4.200 & $1,2 \times 10^{4}$ & 67 \\
\hline
\end{tabular}

$R_{c} \quad$ - Uniaxial compressive strength

$\varepsilon_{1} \quad-$ Longitudinal strain.

E. $=$ Young's modulus tangent.

UMM - Mechanical Microfissuration Threshold.

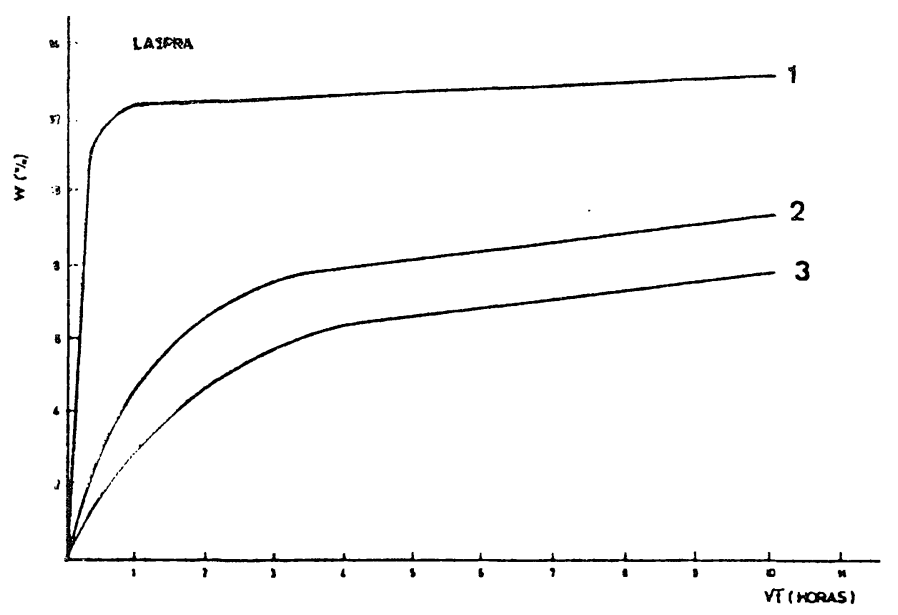

a

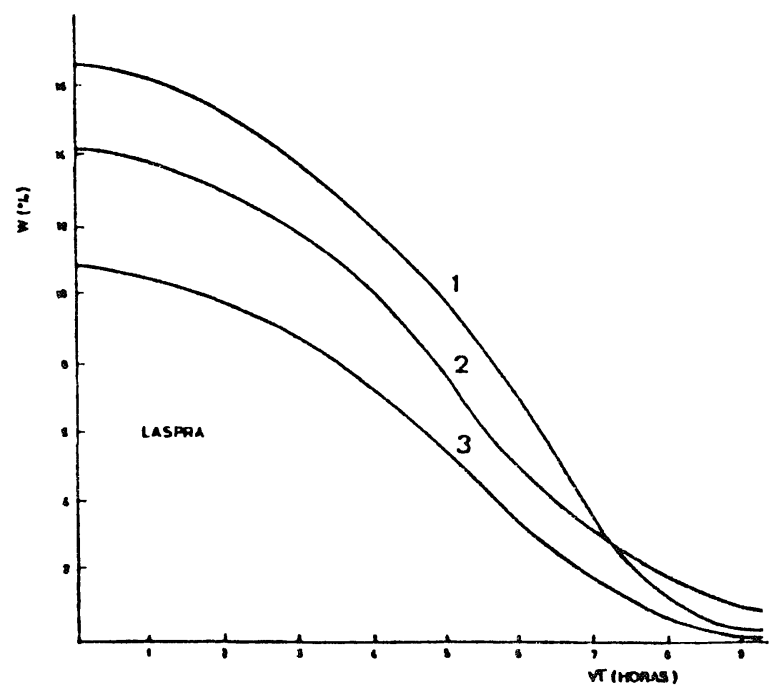

c

Fig. 3.-Curvas de absorción ( $a$ y b) y evaporación (c y d) de agua, en las piedras de Laspra y Piedramuelle. 1 muestras sin tratar; 2 muestras tratadas con RS: 3 muestras tratadas con SE + SO.

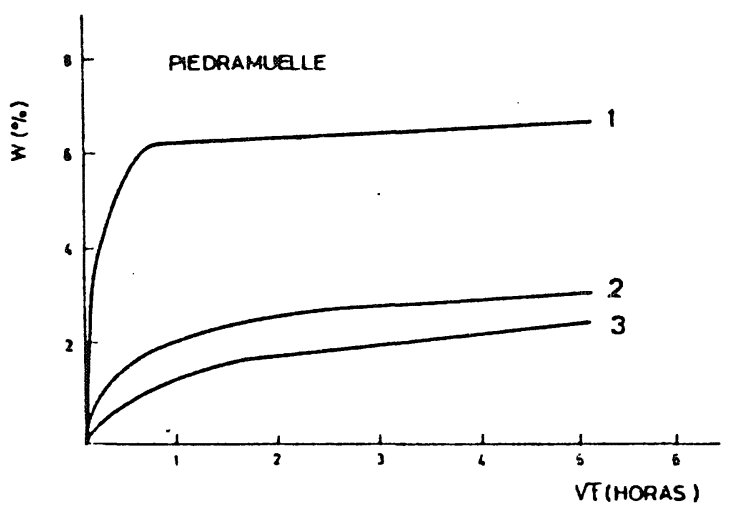

b

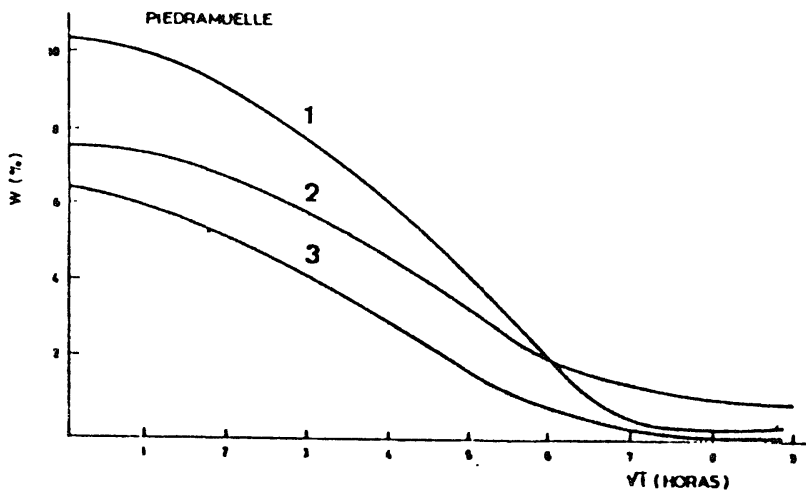

d

Fig. 3.-Absorption curves (a and b), and desorption curves ( $c$ and $d$ ) in the Laspra and Piedramuelle stones. 1: non-treated samples; 2: samples treated with SR: 3 : samples treated with ES and OS.

MATERIALES DE COSTRUCCION, Vol. 37, n.²06, abril/mayo/junio 1987 


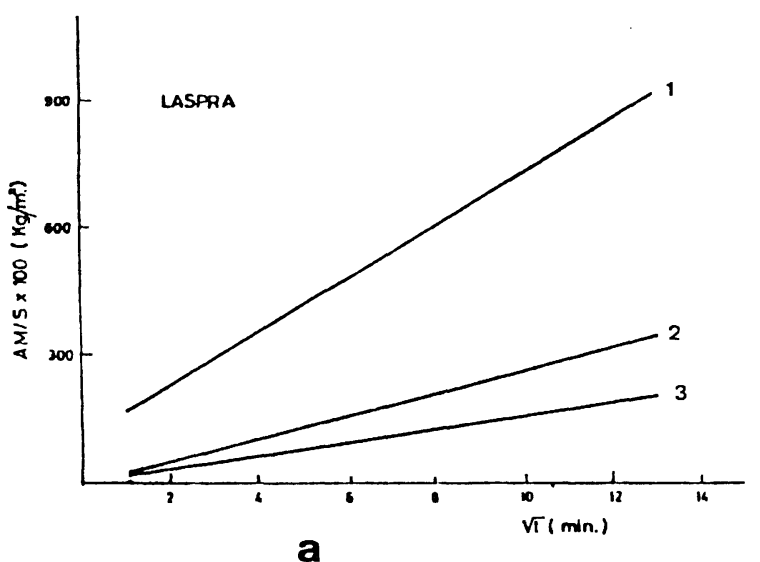

a

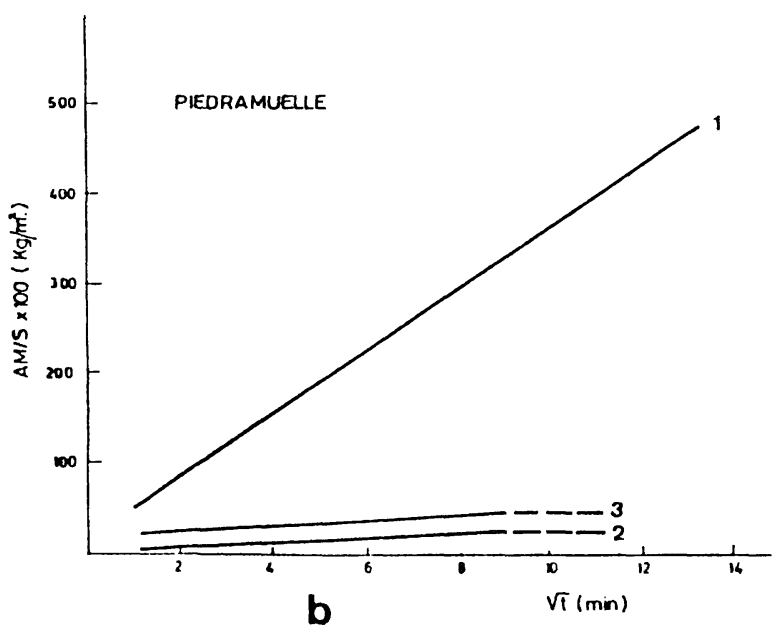

Fig. 4.-Curvas de succión capilar para las piedras de la Catedral, (a) Laspra y (b) Piedramuelle. 1 sin tratar; 2 tratadas con RS y 3 tratadas con SE + SO.

Fig. 4.-Capillary suction curves (a) Laspra, and (b) Piedramuelle. 1: non-treated; 2: treated with SR; 3: treated with ES and OS.

\section{CONCLUSIONES}

- Las propiedades de los materiales y, en especial, aquellas ligadas a los movimientos del agua en el seno de los mismos, varían de los materiales tratados a los no tratados. Puede afirmarse que, en general, se detecta:

- Una disminución de la porosidad abierta.

- Una disminución de la capacidad de absorción de agua por inmersión.
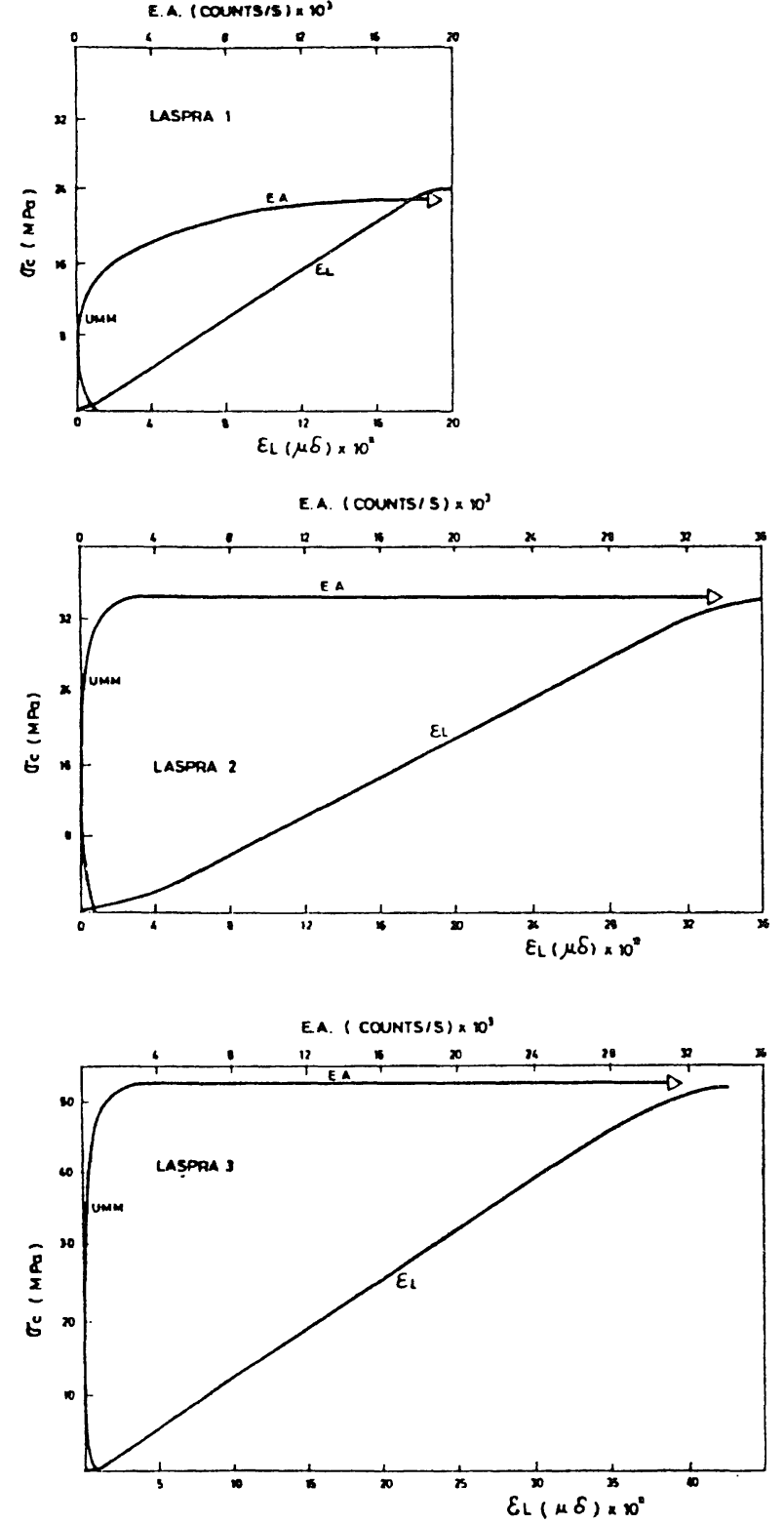

Fig. 5.-Relaciones entre resistencia a la compresión uniaxial $\left(\sigma_{c}\right)$, deformación longitudinal $\left(\varepsilon_{l}\right)$ y emisión acústica en la dolomía de Laspra.

Fig. 5.-Relationship among uniaxial compressive strength $\left(\sigma_{c}\right)$, longitudinal deformation $\left(\varepsilon_{l}\right)$, and acoustic emission in the Laspra Dolomite.

\section{CONCLUSIONS}

The physical properties of the materials, and especially those linked to water movements through the stone, vary from treated to non-treated ones. Generally speaking speaking, we can state:

- A decrease in open porosity.

- A decrease in water absorption by immersion. 
- Una disminución de la succión capilar de agua.

- Una disminución del ritmo de evaporación de agua.

Estas variaciones en las propiedades físicas suponen, en líneas generales, un mejoramiento de las mismas desde el punto de vista de la absorción y penetración de agua en el interior de los materiales tratados; pero por otra parte también indican, que una vez producida la penetración de agua, ésta se evapora con mayor dificultad.

Sin embargo, dichas propiedades difieren de un tipo de piedra a otro y según el tratamiento aplicado, como se desprende de las Figs. 3 y 4.

- Los estudios porométricos ponen de manifiesto que:

Mientras que con el tratamiento con resina silicónica, la distribución porométrica es bastante similar para las muestras tratadas y no tratadas, en el caso del tratamiento con silicato de etilo y silano oligomérico, esta distribución difiere bastante (Laurenzi Tabasso y Santamaría, 1985); en especial en la dolomía de Laspra; localizándose la porosidad de las muestras tratadas, en un rango de radio de acceso de poro de menor tamaño que en las no tratadas (Fig. 2).

- Las propiedades mecánicas también varían al someter las piedras a tratamiento. Así, tomando como ejemplo la dolomía de Laspra, se ha corroborado que su resistencia a la compresión uniaxial aumenta con la aplicación tanto de uno como de otro tratamiento. Su comportamiento deformacional es prácticamente elástico, antes y después del tratamiento, aunque en las muestras tratadas el módulo de Young tangente disminuye, debido a un aumento de la deformabilidad de la roca.

- Por último, se puede observar como el umbral de microfisuración mecánica varía de las muestras sin tratar (UMM $=50 \%)$ a las tratadas, situándose en éstas, más próximo al valor de la rotura final (UMM $=75 \%$ en el caso del tratamiento con la resina silicónica y $U M M=67 \%$ en el caso del tratamiento con silicato de etilo y silano oligomérico) (Tabla III, Fig. 5); siendo las diferencias mínimas entre uno y otro tratamiento.
- A decrease in water capillary suction.

- A decrease in water evaporation.

These variations imply, mainly, their improvement as regards absorption and water penetration in the interior of the materials under treatment; however, they also shows that, once water has permeated, it evaporates with greater difficulty.

Nevertheless, the above mentioned properties differs for each type of rock and treatment employed, as it is shown in Figs. 3 and 4.

On the other hand, porometric studies reveals that; while in the treatment with silicon resin porometric distribution is rather similar for treated and non-treated samples, in the case of ethyl silicate-oligomeric silane treatment, the distribution differs a great deal (Laurenzi Tabasso and Santamaria, 1985), especially in Laspra dolomite. Porosity in treated samples is found in access ranges rank of lesser size that of non-treated samples (Fig. 2).

Mechanical properties also vary when stones undergo treatment. Thus, we have ascertained, for instance, that Laspra dolomite endurance to uniaxial compression strength increases when treated. Their deformational behaviour is practically elastic before and after the treatment, although in the samples treated, Young's modulus decreases due to the increment of rock deformability.

Finally, it can be observed that the mechanical microfissuration threshold varies from non-treated samples (MMT $=50 \%$ ) to the treated ones, these latter being nearer to final rupture values $(M M T=75 \%$ in the case of silicon resin treatment, and $M M T=67 \%$ in the case of ethyl silicate-oligomeric silane) (Table III, Fig. 5). Differences between both treatments are minimal. 


\section{AGRADECIMIENTOS}

A la Comision Asesora de Investigación Científica y Técnica (CAICYT), Proyecto 2573/83 y a la Fundación Ramón Areces, por la financiación de estos temas de investigación.

Al Istituto Centrale del Restauro (Roma) y en especial a la Dra. Marisa L. Tabasso y a Dña. Giuseppina Fazio, por su valiosa ayuda y colaboración en las recomendaciones de los productos y en la puesta a punto de su aplicación.

\section{AKNOWLEDGEMENTS}

The autohors wish to acknowledge to the Scientific and Technical Research Advising Commitee ("Comisión Asesora de Investigación Científica y Técnica -CAICYTProject 2573/83) and the Ramón Areces Foundation, for the financing of this research program. Also to Istituto Centrale del Resturo (Rome). and especially to Dra. Marisa Laurenzzi Tabasso and Mrs. Giuseppina Fazio, for their most valuable help and colaboration in the selection of products and the setting for their use.

\section{BIBLIOGRAFIA}

\section{REFERENCES}

AMOROSO, G. G. y FASSINA V. (1983): "Stone decay and conservation. Atmospheric pollution, cleaning, consolidation and protection". Materials Science Monographs, 11. Elsevier (Ed.) 453 p.

C.N.R.-I.C.R. (Centro di Studio Cause di Deperimento e Metodi de Conservazione delle Opere d'Arte. Istituto Centrale del Restauro) (1981): "Assorbimento d'acqua per immersione totale. Capacità di imbibizione". Doc. Normal: 7/81, Roma, 5 p.

C.N.R.-I.C.R. (1983): "Assorbimento d'acqua per capillarità. Coefficiente di assorbimento capillare". Doc. Normal: 11/82, Roma, $6 \mathrm{p}$.

ESBERT, R. M.; GROSSI, C. y MARCOS, R. M. (1987): "Aplicación de consolidantes y protectores en materiales pétreos de edificación". 1. Semana de la Calidad en la Edificación. Oviedo (en prensa).

ESBERT, R. M.; MARCOS, R. M. y ALONSO, J. (1982): "La deterioración de las piedras de la Catedral de Oviedo. $1 .^{a}$ Parte: Petrografía y porosidad características intrínsecas determinantes de la deterioración de sus rocas carbonatadas". Materiales de Construcción n. 185. Instituto Eduardo Torroja, Madrid, pp. 61-68.

ESBERT, R. M. y MARCOS R. M. (1983): "Las piedras de la Catedral de Oviedo y su deterioración”. Pbl. Colegio Oficial de aparejadores y Arquitectos Técnicos de Asturias. Gráficas Summa, Oviedo, $143 p$

GROSSI, C. (1986): "Ensayos de tratamiento en materiales rocosos de la Catedral de Oviedo". Tesis de Licenciatura. Dpto. de Petrología y Geoquímica. Universidad de Oviedo, $122 \mathrm{p}$.

I.S.R.M. (International Society for Rocks Mechanics) (1979): "Suggested methods for determining: water content, porosity, density, absorption and related properties". Commission on Standarization of Laboratory and Field Test. Int. J. Rock. Mech. Min. Sci \& Geomech. Abst., Vol. 16. n. 2, Pergamon Press, pp. 143-156.

I.S.R.M. (1981): "Rock characterization testing and monitoring". I.S.R.M. suggested methods. E. T. Brown (Ed.), 211 p.

LAURENZI TABASSO, M. y SANTAMARIA, U. (1985): "Consolidant and protective effects of different products on Lecce limestone". $V$ International Congress on Deterioration and Conservartion of Stone. Presses Politechniques Romandes, Lausanne, pp. 697-707.

La II parte de este artículo se publicará en el n. 208 (último n. ${ }^{\circ}$ del presente año). 